\title{
Extrapericardial cardiac tamponade due to massive retrosternal hematoma
}

\author{
Fernando Sabatel-Perez, Miguel A. Sastre-Perona, \\ Mario Baquero Alonso, Luis Rodríguez Padial \\ Department of Cardiology, Hospital Virgen de la Salud, Toledo, Spain
}

A 75-year-old female with a history of mechanical aortic and mitral valves, underwent aortic valve replacement due to dysfunction. During the fourth day after surgery, the patient developed sudden dyspnea at rest and chest discomfort. A physical examination exhibited jugular ingurgitation (Fig. 1A), tachycardia and hypotension. An emergent transthoracic echocardiography revealed a mass close to the right atrium (Fig. 1B, arrow). Because of the said finding, a computed tomography scan was performed which showed an $11 \times$ $\times 9 \mathrm{~cm}$ retrosternal mass that was compressing the right cavities and posterior displacement of the heart (Fig. 1C, D, asterisk). She was brought back to surgery, where a massive retrosternal hematoma was discovered (Fig. 1E, F) with minimal active bleeding caused by three sternal suture points.
After evacuating the hematoma and controlling the bleeding, the patient had an uneventful recovery.

Retrosternal hematoma is a life-threatening condition, because it can result in an extrapericardial cause of cardiac tamponade, induced by heart compression. The most frequent etiologies are traumatic and post-surgical. Given the nonspecificity of the signs and symptoms, a differential diagnosis between classic cardiac tamponade and this entity can be a challenge. Transthoracic echocardiography is the first imaging test for a differential diagnosis. A thoracic computed tomography scan can be helpful when the diagnosis is unclear and also allows rapid detection of active bleeding in some cases. Treatment consists of surgical removal of hematoma and also to control the bleeding, if present.

Conflict of interest: None declared

Address for correspondence: Dr. Fernando Sabatel-Perez, MD, Department of Cardiology, Hospital Virgen de la Salud, Avenida de Barber 30, 45004, Toledo, Spain, tel: +34 9252692 00, e-mail: fernandosabatelperez@gmail.com 

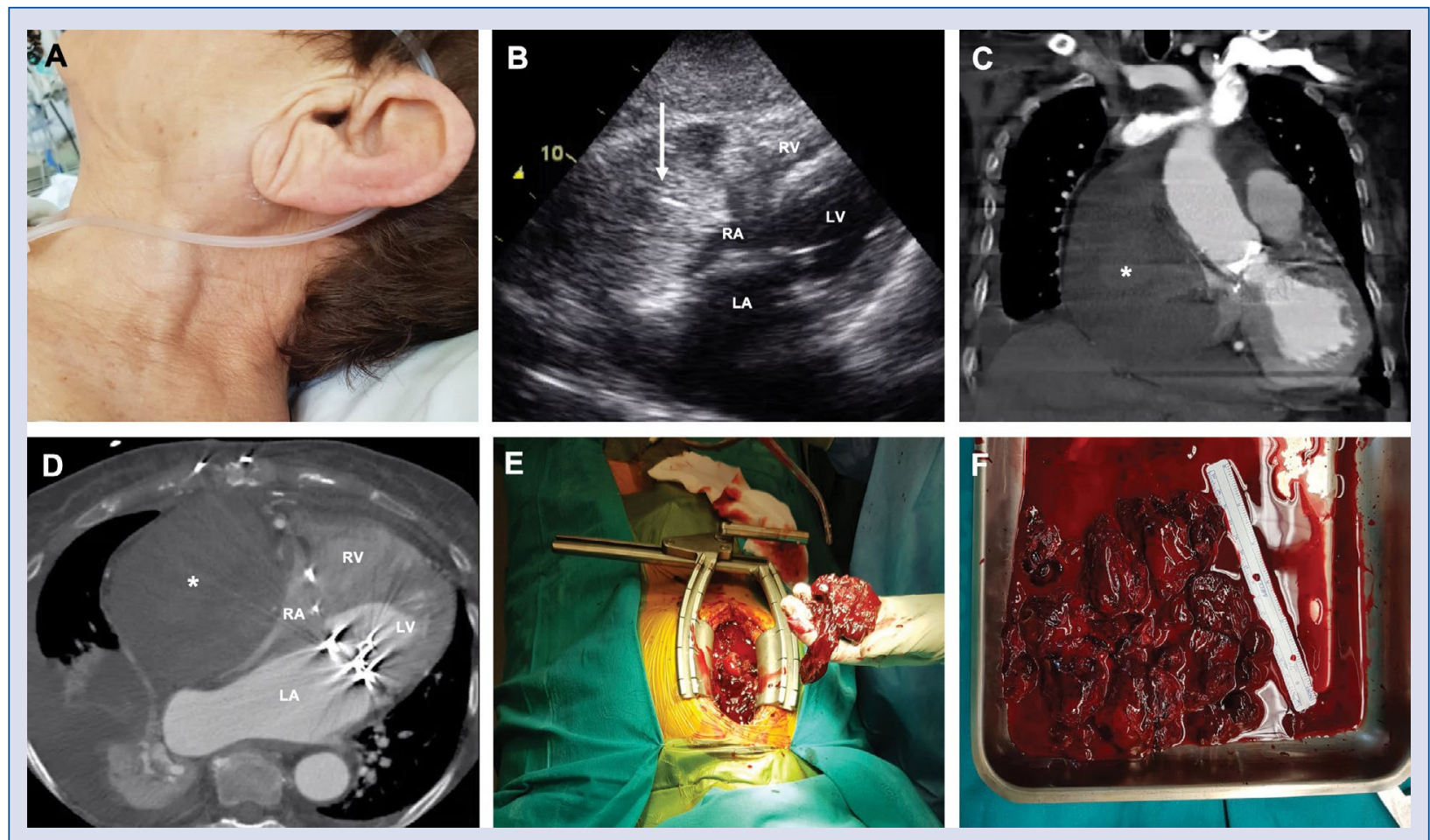

Figure 1. A. Jugular ingurgitation evidenced during physical examination; B. Transthoracic echocardiography, subcostal view. An echogenic mass close to the right atrium is observed (white arrow); LA — left atrium; LV — left ventricle; RA - right atrium; RV — right ventricle; C. Computed tomography scan, frontal axis, where a massive mass to the right of the left ventricle can be appreciated (white asterisk); D. Computed tomography scan, horizontal axis. The mass (white asterisk), compressing right cavities; $\mathbf{E}$. The hematoma removed, in the hands of a surgeon; F. The hematoma removed. 